\title{
Layouts of Graph Subdivisions ${ }^{\star}$
}

\author{
Vida Dujmović ${ }^{1,2}$ and David R. Wood ${ }^{2,3}$ \\ 1 School of Computer Science, McGill University, Montréal, Canada \\ vida@cs.mcgill.ca \\ 2 School of Computer Science, Carleton University, Ottawa, Canada \\ davidw@scs. carleton.ca \\ 3 Department of Applied Mathematics, Charles University, Prague, Czech Republic
}

\begin{abstract}
A $k$-stack layout (respectively, $k$-queue layout) of a graph consists of a total order of the vertices, and a partition of the edges into $k$ sets of non-crossing (non-nested) edges with respect to the vertex ordering. A $k$-track layout of a graph consists of a vertex $k$-colouring, and a total order of each vertex colour class, such that between each pair of colour classes no two edges cross. The stack-number (respectively, queue-number, track-number) of a graph $G$, denoted by $\operatorname{sn}(G)(\operatorname{qn}(G)$, $\operatorname{tn}(G)$ ), is the minimum $k$ such that $G$ has a $k$-stack ( $k$-queue, $k$-track) layout. This paper studies stack, queue, and track layouts of graph subdivisions. It is known that every graph has a 3-stack subdivision. The best known upper bound on the number of division vertices per edge in a 3 -stack subdivision of an $n$-vertex graph $G$ is improved from $\mathcal{O}(\log n)$ to $\mathcal{O}(\log \min \{\operatorname{sn}(G), \mathbf{q n}(G)\})$. This result reduces the question of whether queue-number is bounded by stack-number to whether 3-stack graphs have bounded queue number. It is proved that every graph has a 2queue subdivision, a 4-track subdivision, and a mixed 1-stack 1-queue subdivision. All these values are optimal for every non-planar graph. In addition, we characterise those graphs with $k$-stack, $k$-queue, and $k$-track subdivisions, for all values of $k$. The number of division vertices per edge in the case of 2-queue and 4 -track subdivisions, namely $\mathcal{O}(\log$ qn $(G))$, is optimal to within a constant factor, for every graph $G$. Applications to $3 \mathrm{D}$ polyline grid drawings are presented. For example, it is proved that every graph $G$ has a $3 \mathrm{D}$ polyline grid drawing with the vertices on a rectangular prism, and with $\mathcal{O}(\log \mathrm{qn}(G))$ bends per edge.
\end{abstract}

\section{Introduction}

This paper studies stack, queue and track layouts of subdivisions of graphs. The contributions of this paper are three-fold. First, we characterise those graphs admitting $k$-stack, $k$-queue or $k$-track subdivisions, for all $k$. In addition, we prove bounds on the number of division vertices per edge that are asymptotically tight in a number of cases. These results are presented in Section 3. Second, we use these subdivision layouts to reduce two of the major open problems in the theory of stack and queue layouts to certain special cases. These results, along with relationships amongst various thickness parameters, are presented in

\footnotetext{
* Research supported by NSERC and COMBSTRU. 
Section 4. As the third contribution, we apply our results concerning track layouts of subdivisions to the study of three-dimensional polyline graph drawings. These results are presented in Section 5. Due to space limitations, many proofs and some references are omitted - see [7] for all the details. All logarithms are base 2 unless stated otherwise.

\section{Preliminaries}

We consider undirected, finite, and simple graphs $G$ with vertex set $V(G)$ and edge set $E(G)$. The number of vertices and edges of $G$ are respectively denoted by $n=|V(G)|$ and $m=|E(G)|$. A subdivision of $G$ is a graph obtained from $G$ by replacing each edge $v w \in E(G)$ by a path with at least one edge whose endpoints are $v$ and $w$. Internal vertices on this path are called division vertices. Let $G^{\prime}$ be the subdivision of $G$ with one division vertex per edge.

A graph parameter is a function $\alpha$ that assigns to every graph $G$ a nonnegative integer $\alpha(G)$. Let $\mathcal{G}$ be a class of graphs. By $\alpha(\mathcal{G})$ we denote the function $f: \mathbb{N} \rightarrow \mathbb{N}$, where $f(n)$ is the maximum of $\alpha(G)$, taken over all $n$-vertex graphs $G \in \mathcal{G}$. We say $\mathcal{G}$ has bounded $\alpha$ if $\alpha(\mathcal{G}) \in \mathcal{O}(1)$. A graph parameter $\alpha$ is bounded by a graph parameter $\beta$ (for some class $\mathcal{G}$ ), if there exists a binding function $g$ such that $\alpha(G) \leq g(\beta(G))$ for every graph $G$ (in $\mathcal{G}$ ). If $\alpha$ is bounded by $\beta$ (in $\mathcal{G}$ ) and $\beta$ is bounded by $\alpha$ (in $\mathcal{G}$ ) then $\alpha$ and $\beta$ are tied (in $\mathcal{G}$ ).

A vertex ordering of a graph $G$ is a total order $\sigma$ of the vertex set $V(G)$. Let $L(e)$ and $R(e)$ denote the endpoints of each edge $e \in E(G)$ such that $L(e)<_{\sigma}$ $R(e)$. Consider two edges $e, f \in E(G)$ with no common endpoint such that $L(e)<_{\sigma} L(f)$. If $L(e)<_{\sigma} L(f)<_{\sigma} R(e)<_{\sigma} R(f)$ then $e$ and $f$ cross, and if $L(e)<_{\sigma} L(f)<_{\sigma} R(f)<_{\sigma} R(e)$ then $e$ and $f$ nest. A stack (respectively, queue) is a set of edges $E^{\prime} \subseteq E(G)$ such that no two edges in $E^{\prime}$ cross (nest). Observe that when traversing the vertex ordering, edges in a stack (queue) appear in LIFO (FIFO) order - hence the names. A $k$-stack (queue) layout of $G$ consists of a vertex ordering $\sigma$ of $G$ and a partition $\left\{E_{\ell}: 1 \leq \ell \leq k\right\}$ of $E(G)$, such that each $E_{\ell}$ is a stack (queue) in $\sigma$. A graph admitting a $k$-stack (queue) layout is called a $k$-stack (queue) graph. The stack-number of a graph $G$, denoted by $\operatorname{sn}(G)$, is the minimum $k$ such that $G$ is a $k$-stack graph. The queue-number of a graph $G$, denoted by $\operatorname{qn}(G)$, is the minimum $k$ such that $G$ is a $k$-queue graph. For a summary of results regarding stack and queue layouts see [8].

A vertex $t$-colouring of a graph $G$ is a partition $\left\{V_{i}: 1 \leq i \leq t\right\}$ of $V(G)$ such that for every edge $v w \in E(G)$, if $v \in V_{i}$ and $w \in V_{j}$ then $i \neq j$. Suppose that $<_{i}$ is a total order of each colour class $V_{i}$. Then the pair $\left(V_{i},<_{i}\right)$ is called a track, and $\left\{\left(V_{i},<_{i}\right): 1 \leq i \leq t\right\}$ is a t-track assignment of $G$. We denote track assignments by $\left\{V_{i}: 1 \leq i \leq t\right\}$ when the ordering on each colour class is implicit. An $X$-crossing in a track assignment consists of two edges $v w$ and $x y$ such that $v<_{i} x$ and $y<_{j} w$, for distinct colours $i$ and $j$. A $(k, t)$-track layout of $G$ consists of a $t$-track assignment of $G$ and a (non-proper) edge $k$-colouring of $G$ with no monochromatic X-crossing. $(1, t)$-track layouts (that is, with no $\mathrm{X}$-crossing) are of particular interest due to applications in three-dimensional 
graph drawing (see Section 5). A $(1, t)$-track layout is called a t-track layout. A graph admitting a $t$-track layout is called a $t$-track graph. The track-number of $G$, denoted by $\operatorname{tn}(G)$, is the minimum $t$ such that $G$ is a $t$-track graph. For a summary of bounds on the track-number see [6].

\section{Layouts of Subdivisions}

Stack and queue layouts of graph subdivisions are a central topic of this paper. That every graph has a 3-stack subdivision has been observed by many authors $[10,17,11,1]$. Note that 3 -stack layouts are important in complexity theory, and 3-stack layouts of knots and links, so called Dynnikov digrams, have also recently been considered (see the references in [7]). It is interesting to determine the minimum number of division vertices in a 3-stack subdivision of a given graph. The previously best known bounds are due to Enomoto and Miyauchi [10], who proved that every graph has a 3 -stack subdivision with $\mathcal{O}(\log n)$ division vertices per edge. Moreover, Enomoto et al. [12] proved that this bound is tight up to a constant factor for $K_{n}$ (and some slightly more general families). Thus Enomoto et al. [12] claimed that the $\mathcal{O}(\log n)$ upper bound is 'essentially best possible'. We prove the following refinement of the upper bound of Enomoto and Miyauchi [10], in which the number of division vertices per edge depends on the stack-number or queue-number of the given graph. Moreover, we characterise those graphs admitting $k$-stack subdivisions for all $k$.

Theorem 1. (a) Every graph $G$ has a 3-stack subdivision with $\mathcal{O}(\log \min \{\operatorname{sn}(G), \mathrm{qn}(G)\})$ division vertices per edge.

(b) A graph has a 2-stack subdivision if and only if it is planar. Every planar graph has a 2-stack subdivision with at most one division vertex per edge.

(c) A graph has a 1-stack subdivision if and only if it is outerplanar. Every outerplanar graph has a 1-stack layout (with no division vertices).

Proof Outline. Let $H$ be the subdivision of $G$ with $2\lceil\log \operatorname{sn}(G)\rceil-2$ division vertices per edge. As illustrated in Figure 1, we now prove that $H$ has a 3 -stack subdivision. Consider a $\operatorname{sn}(G)$-stack layout of $G$. Let $T$ be the complete binary tree of height $\lceil\log \mathrm{sn}(G)\rceil$. Consider each stack of $G$ to correspond to a distinct leaf of $T$. Now define a mapping of the vertices of $H$ into the nodes of $T$ such that adjacent vertices of $H$ are mapped to adjacent nodes of $T$ or to the same leaf of $T$. In particular, the original vertices of $G$ are mapped to the root, and each subdivided edge $e$ is mapped to a walk from the root to the leaf corresponding to the stack containing $e$, and then back to the root. A depth-first ordering of $V(T)$ gives a 3 -stack layout of $T$ in which edges with a common endpoint are in distinct stacks. From this layout of $T$ we can obtain the desired 3-stack layout of $H$ by appropriately ordering the vertices of $H$ that are mapped to a single node of $T$, and by assigning each edge $e$ of $H$ to the same stack as the edge of $T$ that $e$ is mapped to. The proof that $G$ has a 3 -stack subdivision with $\mathcal{O}(\log q \mathrm{qn}(G))$ division vertices per edge is similar. Parts (b) and (c) are easy extensions of known results. 


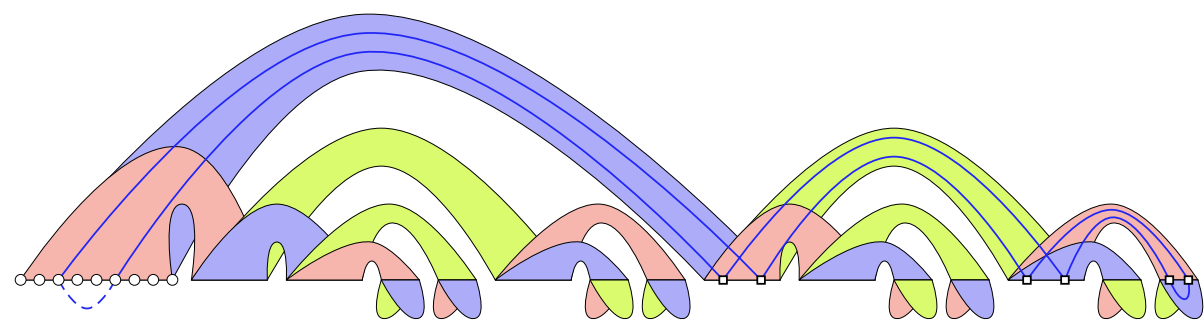

Fig. 1. 3-stack subdivision of a 16-stack graph; one edge is indicated.

Since $\operatorname{sn}(G)$ and $\mathrm{qn}(G)$ are both no more than $n$, our bound in Theorem 1(a) is at most the $\mathcal{O}(\log n)$ bound of Enomoto and Miyauchi [10] (ignoring constant factors). We prove the following analogous result for queue layouts, in which, additionally, the number of division vertices per edge is optimal.

Theorem 2. (a) Every graph $G$ has a 2-queue subdivision with $\mathcal{O}(\log \mathrm{qn}(G))$ division vertices per edge, and every 2-queue subdivision of $G$ has an edge with $\Omega(\log \mathrm{qn}(G))$ division vertices per edge.

(b) A graph has a 1-queue subdivision if and only if it is planar.

Thus, at least for the representation of graph subdivisions, two queues suffice rather than three stacks. In this sense, queues are more powerful than stacks. We have the following analogous result for track layouts.

Theorem 3. (a) Every graph $G$ has a 4-track subdivision with $\mathcal{O}(\log \mathrm{qn}(G))$ division vertices per edge, and every 4-track subdivision of $G$ has an edge with $\Omega(\log \mathrm{qn}(G))$ division vertices.

(b) A graph has a 3-track subdivision if and only if it is planar.

(c) A graph has a 2-track subdivision if and only if it is a forest of caterpillars.

A trade-off between the number of stacks and the number of division vertices in 3-stack subdivisions was observed by Enomoto and Miyauchi [11], who proved that for all $s \geq 3$, every graph has an $s$-stack subdivision with $\mathcal{O}\left(\log _{s-1} n\right)$ division vertices per edge. Again Enomoto et al. [12] proved that this bound is tight up to a constant factor for $K_{n}$. As described in Table 1 , our results for 3 -stack subdivisions, 2-queue subdivisions, and 4-track subdivisions generalise in a similar fashion to the result of Enomoto and Miyauchi [11]. Moreover, we

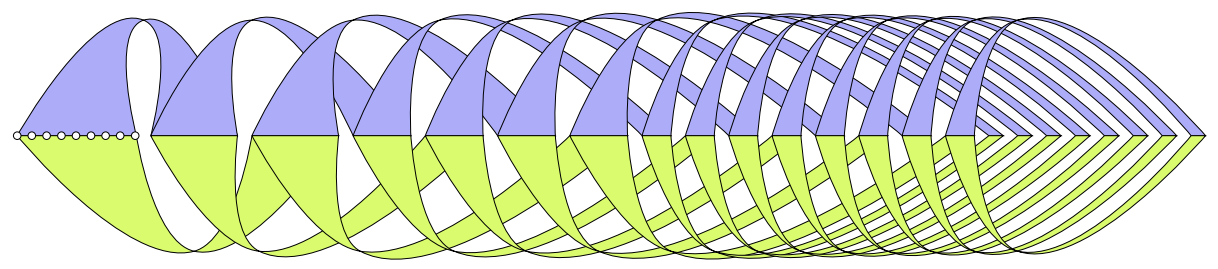

Fig. 2. A 2-queue subdivision of an 8-queue graph. 
Table 1. Layouts of a subdivision of a graph $G$.

\begin{tabular}{crcc}
\hline \multicolumn{1}{c}{ graph } & \multicolumn{2}{c}{ type of layout } & \# division vertices per edge \\
\hline arbitrary & \multicolumn{1}{c}{$s$-stack } & $(s \geq 3)$ & $\mathcal{O}\left(\log _{s-1} \operatorname{sn}(G)\right)$ \\
arbitrary & $s$-stack & $(s \geq 3)$ & $\mathcal{O}\left(\log _{s-1}\right.$ qn $\left.(G)\right)$ \\
planar & 2-stack & & 1 \\
\hline arbitrary & q-queue & $(q \geq 2)$ & $\Theta\left(\log _{q}\right.$ qn $\left.(G)\right)$ \\
planar & 1-queue & & $n-2$ \\
\hline arbitrary & $s$-stack $q$-queue & $(s \geq 1, q \geq 1)$ & $\mathcal{O}\left(\log _{(s+q) q}\right.$ sn $\left.(G)\right)$ \\
arbitrary & $s$-stack q-queue & $(s \geq 1, q \geq 1)$ & $\mathcal{O}\left(\log _{(s+q) q}\right.$ qn $\left.(G)\right)$ \\
planar & 1-stack 1-queue & & 4 \\
\hline arbitrary & $(d+1,2)$-track & $(d \geq 2)$ & $\Theta\left(\log _{d}\right.$ qn $\left.(G)\right)$ \\
arbitrary & $(d, 3)$-track & $(d \geq 2)$ & $\Theta\left(\log _{d}\right.$ qn $\left.(G)\right)$ \\
arbitrary & $(d+2)$-track & $(d \geq 2)$ & $\Theta\left(\log _{d}\right.$ qn $\left.(G)\right)$ \\
planar & 3-track & & $n-2$ \\
\hline
\end{tabular}

generalise stack and queue layouts through the notion of a mixed layout. Here each edge is assigned to a stack or to a queue, defined with respect to a common vertex ordering. We speak of an s-stack q-queue mixed layout and an s-stack $q$ queue graph. Part of the motivation for studying mixed stack and queue layouts is that they model the double-ended queue (dequeue) data structure, since a dequeue may be simulated by two stacks and one queue.

\section{Relationships}

The following lemma highlights the fundamental relationship between track layouts, and queue and stack layouts. Its proof follows immediately from the definitions, and is illustrated in Figure 3 for $k=1$.

Lemma 1. Let $\{A, B\}$ be a track assignment of a bipartite graph $G$. Then the following are equivalent:

(a) $\{A, B\}$ admits a $(k, 2)$-track layout of $G$,

(b) the vertex ordering with $A$ followed by $B$ admits a $k$-queue layout of $G$, and

(c) the vertex ordering with $A$ followed by the reversal of $B$ admits a $k$-stack layout of $G$.

The relationship between queue and track layouts in Lemma 1 was extended by Dujmović et al. [6] who proved that queue-number and track-number are tied. Despite a wealth of research on stack and queue layouts, the following fundamental questions of Heath et al. [15] remain unanswered ${ }^{1}$.

\footnotetext{
${ }^{1}$ Heath et al. [15], in their study of the relationship between stack- and queue-number, restricted themselves to linear binding functions. For example, for stack-number to be bounded by queue-number meant that $\operatorname{sn}(G) \in \mathcal{O}(\mathrm{qn}(G))$ for every graph $G$. Thus Heath et al. [15] considered Open Problem 1 to be solved in the negative by

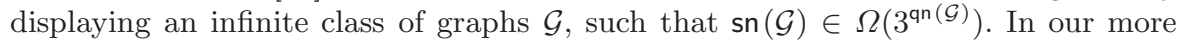
liberal definition of a binding function, this result merely provides a lower bound on a potential binding function.
} 


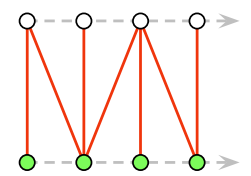

(a)

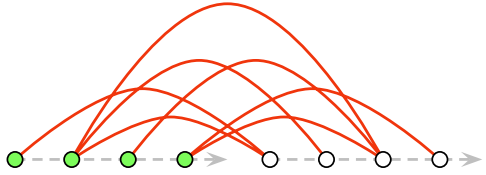

(b)

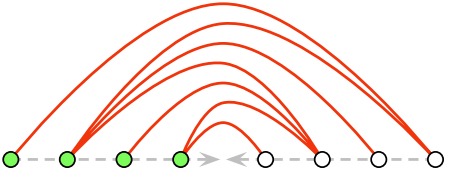

(c)

Fig. 3. Layouts of a caterpillar: (a) 2-track, (b) 1-queue, (c) 1-stack.

Open Problem 1. [15] Is stack-number bounded by queue-number?

Open Problem 2. [15] Is queue-number bounded by stack-number?

Suppose that stack-number is bounded by queue-number, but queue-number is not bounded by stack-number. This would happen, for example, if there exists a constant $s$ such that for every $q$ there exists an $s$-stack graph with no $q$-queue layout. Then we would consider stacks to be more 'powerful' than queues. In the remainder of this section we show that the study of stack, queue and track layouts of subdivisions provides insights into these open problems.

Let $\alpha$ be a graph parameter. Let sub- $\alpha$ be the graph parameter defined by sub- $\alpha(G)=\alpha\left(G^{\prime}\right)$ for every graph $G$. We say $\alpha$ is topological if $\alpha$ and sub- $\alpha$ are tied. For example, chromatic number is not topological since $G^{\prime}$ is bipartite. On the other hand tree-width is topological. In fact, the tree-width of $G$ equals the tree-width of every subdivision of $G$. Similarly crossing number is topological.

The thickness of a graph $G$, denoted by $\theta(G)$, is the minimum number of subgraphs in a partition of $E(G)$ into planar subgraphs. Thickness is not topological since it is easily seen that $\theta\left(G^{\prime}\right) \leq 2$. The geometric thickness of a graph $G$, denoted by $\bar{\theta}(G)$, is the minimum number of colours such that $G$ can be drawn in the plane with edges as coloured straight-line segments, such that monochromatic edges do not cross. Eppstein [13] proved that $\bar{\theta}\left(G^{\prime}\right) \leq 2$ for every graph $G$. Thus geometric thickness is not topological.

Stack-number (or book-thickness) is equivalent to geometric thickness with the additional requirement that the vertices are in convex position. Thus

$$
\forall \text { graph } G, \theta(G) \leq \bar{\theta}(G) \leq \operatorname{sn}(G) .
$$

Blankenship and Oporowski [1], Enomoto and Miyauchi [10], and Eppstein [13] independently proved that $\operatorname{sn}\left(K_{n}\right)$ is bounded by $\operatorname{sn}\left(K_{n}^{\prime}\right)$. The proofs by Blankenship and Oporowski [1] and Eppstein [13] use essentially the same Ramseytheoretic argument. Since $\bar{\theta}\left(K_{n}^{\prime}\right)=2$, Eppstein [13] observed that stack-number is not bounded by geometric thickness. Using a more elaborate Ramsey-theoretic argument, Eppstein [13] proved that geometric thickness is not bounded by thickness. In particular, for every $t$ there exists a graph with thickness three and geometric thickness at least $t$. Blankenship and Oporowski [1] conjecture that their result for complete graphs extends to all graphs.

Conjecture 1. [1] There exists a function $f$, such that for every graph $G$ and every subdivision $H$ of $G$ with at most one division vertex per edge, we have $\operatorname{sn}(G) \leq f(\operatorname{sn}(H))$.

We now prove that Conjecture 1 is related to Open Problem 1. 
Theorem 4. If Conjecture 1 is true then stack-number is topological, and stacknumber is bounded by queue-number.

Proof Outline. Conjecture 1 would imply that sn is bounded by sub-sn, which would imply that stack-number is topological since it is easily seen that $\operatorname{sn}\left(G^{\prime}\right) \leq$ $\mathrm{sn}(G)+1$. It follows from Conjecture 1 that there exists a function $f^{*}$ such that for any $s$-stack subdivision of a graph $G$ with $k$ division vertices per edge, $G$ has a $f^{*}(s, k)$-stack layout. By Theorem $1($ a), every graph $G$ has a 3 -stack subdivision with $\mathcal{O}(\log \mathrm{qn}(G))$ division vertices per edge. Thus sn $(G) \leq f^{*}(3, \mathcal{O}(\log \mathrm{qn}(G)))$, and stack-number is bounded by queue-number.

We now turn our attention to the question of whether queue-number is topological. The next lemma is proved by repeated application of the Erdös-Szekeres Theorem regarding monotone subsequences.

Lemma 2. If a q-queue subdivision of a graph $G$ has at most $k$ division vertices per edge, then $\operatorname{qn}(G) \in \mathcal{O}\left(q^{2 k}\right)$.

Lemma 2 is used to prove the lower bounds on the number of division vertices per edge in Theorem 2(a) and Theorem 3(a). It follows from Lemma 2 that:

Theorem 5. Queue-number is topological (for all graphs), and track-number is topological for any proper minor-closed graph family.

We now relate queue-number to a new thickness parameter. Let the 2-track thickness of a bipartite graph $G$, denoted by $\theta_{2}(G)$, be the minimum $k$ such that $G$ has a $(k, 2)$-track layout. By $(1)$ and Lemma $1(\mathrm{c})$,

$$
\forall \text { bipartite graphs } G, \theta(G) \leq \bar{\theta}(G) \leq \operatorname{sn}(G) \leq \theta_{2}(G) .
$$

Let the 2-track sub-thickness of a graph $G$, denoted by sub- $\theta_{2}(G)$, be the 2-track thickness of $G^{\prime}$. This is well-defined since $G^{\prime}$ is bipartite.

Theorem 6. Queue-number is tied to 2-track thickness for bipartite graphs, and queue-number is tied to 2-track sub-thickness (for all graphs).

Theorem 6 is somewhat counterintuitive since, at first glance, queue layouts may have many crossings, as opposed to the various thickness parameters. The immediate implication for Open Problem 1 is that stack-number is bounded by queue-number if and only if stack-number is bounded by 2-track sub-thickness. While it is an open problem whether stack number is bounded by track-number or by queue-number, in [6] we prove the weaker result that geometric thickness is bounded by track-number, which implies that geometric thickness is bounded by queue-number. We have the following reductions for Open Problem 2.

Theorem 7. The following are equivalent:

(a) queue-number is bounded by stack-number,

(b) bipartite 3-stack graphs have bounded queue-number,

(c) bipartite 3-stack graphs have bounded 2-track thickness.

Moreover, if queue-number is bounded by stack-number then queue-number is bounded by a polynomial function of stack-number. 
Proof Outline. That (a) implies (b) is immediate. Theorem 6 proves that (b) and (c) are equivalent. It remains to prove that (b) implies (a). Suppose that every bipartite 3-stack graph has queue-number at most some constant $q$. Consider an arbitrary graph $G$. An easy extension of Theorem 1(a) proves that $G$ has a 3-stack bipartite subdivision $D$ with $\mathcal{O}(\log \operatorname{sn}(G))$ division vertices per edge. By assumption, $\mathrm{qn}(D) \leq q$. By Lemma 2, and with an abuse of $\mathcal{O}()$ notation, qn $(G) \in \mathcal{O}\left(q^{\mathcal{O}(\log \operatorname{sn}(G))}\right) \in \mathcal{O}\left(\operatorname{sn}(G)^{\mathcal{O}(q)}\right)$. Thus queue-number is bounded by a polynomial function of stack-number.

For Theorem 7 to hold, it is essential that the number of division vertices per edge in Theorem 1(a) is some function of $\operatorname{sn}(G)$, thus emphasising the significance of our bound in comparison with previous results.

\section{Three-Dimensional Polyline Drawings}

A $3 D$ polyline drawing of a graph represents the vertices by distinct points in $\mathbb{Z}^{3}$ (called gridpoints), and represents each edge as a polyline between its endpoints with bends (if any) also at gridpoints, such that distinct edges only intersect at common endpoints, and each edge only intersects a vertex that is an endpoint of that edge. A 3D polyline drawing with at most $b$ bends per edge is called a $3 D$ b-bend drawing. A 3D 0-bend drawing is called a 3D straight-line drawing. Of course, a 3D $b$-bend drawing of a graph $G$ is precisely a 3D straight-line drawing of a subdivision of $G$ with at most $b$ division vertices per edge. The bounding box of a $3 \mathrm{D}$ polyline drawing is the minimum axis-aligned box containing the drawing. If the bounding box has side lengths $X-1, Y-1$ and $Z-1$, then we speak of an $X \times Y \times Z$ polyline drawing with volume $X \cdot Y \cdot Z$. That is, the volume of a $3 \mathrm{D}$ drawing is the number of gridpoints in the bounding box.

This paper initiates the study of upper bounds on the volume and number of bends per edge in arbitrary 3D polyline drawings. The volume of $3 \mathrm{D}$ straightline drawings has been widely studied $[4,3,14,19,2]$. Table 2 summarises the best known upper bounds on the volume and bends per edge, including those established in this paper. Our upper bound of $\mathcal{O}(m \log q)$ is within a factor of $\mathcal{O}(\log q)$ of being optimal for all $q$-queue graphs, since Bose et al. [2] proved that $3 \mathrm{D}$ polyline drawings have at least $\frac{1}{8}(n+m)$ volume.

Track layouts have previously been used to produce 3D drawings with small volume (see [5]). The principle idea is to position the vertices in a single track on a vertical 'rod'. Since there are no X-crossings in the track layout, no edges between the same pair of tracks can cross.

Theorem 8. $[\mathbf{9}, \mathbf{5}]$ Let $G$ be a c-colourable t-track graph. Then

(a) $G$ has a $\mathcal{O}(t) \times \mathcal{O}(t) \times \mathcal{O}(n)$ straight-line drawing with $\mathcal{O}\left(t^{2} n\right)$ volume, and (b) $G$ has a $\mathcal{O}(c) \times \mathcal{O}\left(c^{2} t\right) \times \mathcal{O}\left(c^{4} n\right)$ straight-line drawing with $\mathcal{O}\left(c^{7}\right.$ tn $)$ volume.

Moreover, if $G$ has an $X \times Y \times Z$ straight-line drawing then $G$ has track-number $\operatorname{tn}(G) \leq 2 X Y$.

By Theorem 3(a), every graph has a 4-track subdivision with $\mathcal{O}(\log n)$ division vertices per edge, and hence a $3 \mathrm{D}$ polyline drawing with $\mathcal{O}(n+m \log n)$ volume by Theorem $8(\mathrm{a})$. We have the following specific results. 
Table 2. Volume of 3D polyline drawings of graphs with $n$ vertices and $m \geq n$ edges.

\begin{tabular}{lcll}
\hline graph family & bends per edge & volume & reference \\
\hline arbitrary & 0 & $\mathcal{O}\left(n^{3}\right)$ & Cohen et al. $[3]$ \\
arbitrary & 0 & $\mathcal{O}\left(m^{4 / 3} n\right)$ & Dujmović and Wood [9] \\
maximum degree $\Delta$ & 0 & $\mathcal{O}(\Delta m n)$ & Dujmović and Wood [9] \\
bounded maximum degree & 0 & $\mathcal{O}\left(m^{1 / 2} n\right)$ & Dujmović and Wood [9] \\
bounded chromatic number & 0 & $\mathcal{O}\left(n^{2}\right)$ & Pach et al. $[19]$ \\
bounded chromatic number & 0 & $\mathcal{O}\left(m^{2 / 3} n\right)$ & Dujmović and Wood [9] \\
$H$-minor free $(H$ fixed) & 0 & $\mathcal{O}\left(n^{3 / 2}\right)$ & Dujmović and Wood [9] \\
bounded tree-width & 0 & $\mathcal{O}(n)$ & Dujmović et al. $[5]$ \\
$c$-colourable $q$-queue & 1 & $\mathcal{O}(c q m)$ & Theorem 9(a) \\
arbitrary & 1 & $\mathcal{O}(n m)$ & Theorem 9(b) \\
$q$-queue & 2 & $\mathcal{O}(q n)$ & Theorem 9(c) \\
$q$-queue (constant $\epsilon>0)$ & $\mathcal{O}(1)$ & $\mathcal{O}\left(m q^{\epsilon}\right)$ & Theorem 10 \\
$q$-queue & $\mathcal{O}(\log q)$ & $\mathcal{O}(m \log q)$ & Theorem 12 \\
\hline
\end{tabular}

Theorem 9. Every c-colourable q-queue graph has: (a) a $2 \times c(q+1) \times(n+m)$ polyline 1-bend drawing, (b) an $n \times m \times 2$ polyline 1-bend drawing, and (c) a $2 \times 2 q \times(2 n-3)$ polyline 2 -bend drawing.

The next result highlights the apparent trade-off between few bends and small volume.

Theorem 10. For every $\epsilon>0$, every q-queue graph has a $2 \times \mathcal{O}\left(q^{\epsilon}\right) \times \mathcal{O}(n+m / \epsilon)$ polyline drawing with $\mathcal{O}(1 / \epsilon)$ bends per edge.

Felsner et al. [14] introduced 3D straight-line graph drawings with the vertices positioned on the edges of a triangular or rectangular prism.

Theorem 11. Every planar graph has a $2 \times 2 \times \mathcal{O}\left(n^{2}\right)$ polyline drawing on a triangular prism with at most $n-2$ bends per edge. Only planar graphs have polyline drawings on a triangular prism.

Theorem 12. Every q-queue graph $G$ has a $2 \times 2 \times \mathcal{O}(n+m \log q)$ polyline drawing on a rectangular prism with $\mathcal{O}(\log q)$ bends per edge.

Proof. By Theorem 3(a), $G$ has a 4 -track subdivision $D$ with $\mathcal{O}(\log q)$ division vertices per edge. The number of vertices of $D$ is $\mathcal{O}(n+m \log q)$. Let $\left\{V_{1}, V_{2}, V_{3}, V_{4}\right\}$ be the tracks. Let $n^{\prime}=\max \left\{\left|V_{1}\right|,\left|V_{2}\right|,\left|V_{3}\right|,\left|V_{4}\right|\right\}$. Position the $i^{\text {th }}$ vertex in $V_{1}$ at $(0,0,2 i)$. Position the $i^{\text {th }}$ vertex in $V_{2}$ at $(1,0,2 i)$. Position the $i^{\text {th }}$ vertex in $V_{3}$ at $(0,1,2 i)$. Position the $i^{\text {th }}$ vertex in $V_{4}$ at $(1,1,2 i+1)$. Clearly the only possible crossing is between edges $v w$ and $x y$ with $v \in V_{1}, w \in V_{4}$, $x \in V_{2}$, and $y \in V_{3}$. Such a crossing point is on the line $L=\left\{\left(\frac{1}{2}, \frac{1}{2}, z\right): z \in \mathbb{R}\right\}$. However, $v w$ intersects $L$ at $\left(\frac{1}{2}, \frac{1}{2}, \alpha+\frac{1}{2}\right)$ for some integer $\alpha$, and $x y$ intersects $L$ at $\left(\frac{1}{2}, \frac{1}{2}, \beta\right)$ for some integer $\beta$. Thus $v w$ and $x y$ do not intersect. The bounding box is $2 \times 2 \times 2 n^{\prime}$, which is $2 \times 2 \times \mathcal{O}(n+m \log q)$.

Note that Di Giacomo and Meijer [4] proved that a 4-track graph has a $2 \times 2 \times n$ drawing. When $n^{\prime}<\frac{n}{2}$ the above construction has less volume. 


\section{Planar Graphs}

Felsner et al. [14] asked the following question (in their conference paper).

Open Problem 3. [14] Does every $n$-vertex planar graph have a $3 \mathrm{D}$ straightline drawing with $\mathcal{O}(n)$ volume?

By Theorem 8, this question has an affirmative answer if planar graphs have bounded track-number. Whether planar graphs have bounded track-number is an open problem due to Hubert de Fraysseix [private communication, 2000], and since queue-number is tied to track-number for planar graphs $[5,6]$, is equivalent to the following open problem due to Heath et al. [15]. Note that the best known upper bound on the queue-number of planar graphs is $\mathcal{O}(\sqrt{n})$.

Open Problem 4. [15] Do planar graphs have bounded queue-number?

We make the following contribution to the study of this problem, which is analogous to Theorem 7, since 2-stack graphs are precisely the subgraphs of Hamiltonian planar graphs.

Theorem 13. Let $\mathcal{F}(n)$ be the family of functions $\mathcal{O}(1)$ or $\mathcal{O}(\operatorname{polylog} n)$. The following are equivalent:

(a) n-vertex planar graphs have queue-number in $\mathcal{F}(n)$,

(b) n-vertex bipartite Hamiltonian planar graphs have queue-number in $\mathcal{F}(n)$,

(c) n-vertex bipartite Hamiltonian planar graphs have 2-track thickness in $\mathcal{F}(n)$.

(d) n-vertex planar graphs have $\mathcal{O}(1) \times \mathcal{O}(1) \times \mathcal{O}(n)$ polyline $\mathcal{O}(1)$-bend drawings.

\section{Acknowledgements}

Thanks to Stefan Langerman for stimulating discussions on 3D polyline drawings. Thanks to Franz Brandenburg and Ulrik Brandes for pointing out the connection to double-ended queues. Thanks to Ferran Hurtado and Prosenjit Bose for graciously hosting the second author, whose research was partially completed at the Departament de Matemàtica Aplicada II, Universitat Politècnica de Catalunya, Barcelona, Spain.

\section{References}

1. Robin Blankenship and Bogdan Oporowski. Drawing subdivisions of complete and complete bipartite graphs on books. Technical Report 1999-4, Department of Mathematics, Louisiana State University, 1999.

2. Prosenjit Bose, Jurek Czyzowicz, Pat Morin, and David R. Wood. The maximum number of edges in a three-dimensional grid-drawing. J. Graph Algorithms Appl., 8(1):21-26, 2004.

3. Robert F. Cohen, Peter Eades, Tao Lin, and Frank Ruskey. Threedimensional graph drawing. Algorithmica, 17(2):199-208, 1996.

4. Emilio Di Giacomo and Henk Meijer. Track drawings of graphs with constant queue number. In LiotTA [16], pages 214-225. 
5. Vida Dujmović, Pat Morin, And David R. Wood. Layout of graphs with bounded tree-width. SIAM J. Comput., to appear.

6. Vida Dujmović, Attila Pór, And David R. Wood. Track layouts of graphs. Submitted; see arXiv:cs.DM/0407033, 2004.

7. Vida Dujmović And David R. Wood. Stacks, queues and tracks: Layouts of graph subdivisions. Submitted; see Tech. Rep. TR-2003-08, School of Computer Science, Carleton University, Ottawa, Canada, 2003.

8. Vida Dujmović and David R. Wood. On linear layouts of graphs. Discrete Math. Theor. Comput. Sci., 6(2):339-358, 2004.

9. Vida Dujmović and David R. Wood. Three-dimensional grid drawings with sub-quadratic volume. In $\mathrm{PACH}$ [18], pages 55-66.

10. Hikoe Enomoto and Miki Shimabara Miyauchi. Embedding graphs into a three page book with $O(M \log N)$ crossings of edges over the spine. SIAM J. Discrete Math., 12(3):337-341, 1999.

11. Hikoe Enomoto and Miki Shimabara Miyauchi. Embedding a graph into a $d+1$-page book with $\left\lceil m \log _{d} n\right\rceil$ edge-crossings over the spine. IPSJ SIGNotes ALgorithms, 051, 2001. Abstract No. 008.

12. Hikoe Enomoto, Miki Shimabara Miyauchi, and Katsuhiro Ota. Lower bounds for the number of edge-crossings over the spine in a topological book embedding of a graph. Discrete Appl. Math., 92(2-3):149-155, 1999.

13. David Eppstein. Separating thickness from geometric thickness. In PACH [18], pages $75-86$.

14. Stefan Felsner, Giussepe Liotta, and Stephen Wismath. Straight-line drawings on restricted integer grids in two and three dimensions. J. Graph Algorithms Appl., 7(4):363-398, 2003.

15. Lenwood S. Heath, Frank Thomson Leighton, and Arnold L. Rosenberg. Comparing queues and stacks as mechanisms for laying out graphs. SIAM J. Discrete Math., 5(3):398-412, 1992.

16. Guiseppe Liotta, editor. Proc. 11th International Symp. on Graph Drawing (GD '03), volume 2912 of Lecture Notes in Comput. Sci. Springer, 2004.

17. Miki Shimabara Miyauchi. An $O(n m)$ algorithm for embedding graphs into a 3-page book. Trans. IEICE, E77-A(3):521-526, 1994.

18. JÁnos PACH, editor. Towards a Theory of Geometric Graphs, volume 342 of Contemporary Mathematics. Amer. Math. Soc., 2004.

19. János Pach, Torsten Thiele, And GézA Tóth. Three-dimensional grid drawings of graphs. In Bernard Chazelle, Jacob E. Goodman, And Richard POLLACK, editors, Advances in discrete and computational geometry, volume 223 of Contemporary Mathematics, pages 251-255. Amer. Math. Soc., 1999. 